\title{
On a New Class of $p$-Valent Meromorphic Functions Defined in Conic Domains
}

\section{Mohammed Ali Alamri and Maslina Darus}

School of Mathematical Sciences, Faculty of Science and Technology, Universiti Kebangsaan Malaysia, 43600 Bangi, Selangor, Malaysia

Correspondence should be addressed to Maslina Darus; maslina@ukm.edu.my

Received 26 April 2016; Accepted 27 June 2016

Academic Editor: Jin-Lin Liu

Copyright (C) 2016 M. A. Alamri and M. Darus. This is an open access article distributed under the Creative Commons Attribution License, which permits unrestricted use, distribution, and reproduction in any medium, provided the original work is properly cited.

We define a new class of multivalent meromorphic functions using the generalised hypergeometric function. We derived this class related to conic domain. It is also shown that this new class of functions, under certain conditions, becomes a class of starlike functions. Some results on inclusion and closure properties are also derived.

\section{Introduction}

Let $M_{p}$ denote the class of functions of the form

$$
f(z)=\frac{1}{z^{p}}+\sum_{n=1}^{\infty} a_{n} z^{n-p}, \quad p \in \mathbb{N}=1,2,3, \ldots,
$$

which are analytic and $p$-valent in the punctured unit disc centred at origin $E^{*}=\{z: 0<|z|<1\}=E \backslash\{0\}$. Also by $f(z) \prec g(z)$ we mean $f(z)$ is subordinate to $g(z)$ which implies the existence of an analytic function, called Schwartz function $w(z)$ with $|w(z)|<1$, for $z \in E^{*}$ such that $f(z)=g(w(z))$, where $f(z)$ and $g(z)$ are multivalent meromorphic functions. Note that if $g$ is univalent in $E$ then the above subordination is equivalent to $f(0)=g(0)$ and $f(E) \subset g(E)$.

The set of points, for $0<\gamma<1$ and $k \in[0, \infty)$,

$$
\Omega_{k, \gamma}=\gamma \Omega_{k}+(1-\gamma) \text {, }
$$

where

$$
\Omega_{k}=\left\{u+i v: u>k \sqrt{(u-1)^{2}+v^{2}}, u>0\right\},
$$

[1] showed that the extremal functions $q_{k, \gamma}(z)$ for conic regions are convex univalent and given by

$$
q_{k, \gamma}(z)= \begin{cases}\frac{1+(1-2 \gamma) z}{1-z}, & k=0, \\ 1+\frac{2 \gamma}{1-k^{2}}\left[\frac{2}{\pi}(\arccos k) \operatorname{arctanh} \sqrt{z}\right], & 0<k<1, \\ 1+\frac{2 \gamma}{\pi^{2}}\left(\log \frac{1+\sqrt{z}}{1-\sqrt{z}}\right)^{2}, & k=1, \\ 1+\frac{\gamma}{k^{2}-1}\left[\sin \left(\frac{\pi}{2 R(t)} \int_{0}^{u(z) / \sqrt{t}} \frac{1}{\sqrt{1-x^{2}} \sqrt{1-(t x)^{2}}} \mathrm{~d} x\right)-1\right], & k>1,\end{cases}
$$

where $R(t)$ is Legendre's complete elliptic integral of the first kind with $R^{\prime}(t)=\sqrt{1-t^{2}}$ as its complementary integral, $u(z)=(z-\sqrt{t}) /(1-\sqrt{t} z), t \in(0,1)$, and $z \in E$ is chosen in such a way that $k=\cosh \left(\pi R^{\prime}(t) / R(t)\right)$. 
The generalised hypergeometric function ${ }_{q} F_{s}\left(\alpha_{1}, \ldots, \alpha_{q}\right.$; $\left.\beta_{1}, \ldots, \beta_{s} ; z\right)$ for complex parameters $\alpha_{1}, \ldots, \alpha_{q}$ and $\beta_{1}, \ldots, \beta_{s}$ with $\beta_{j} \neq 0,-1,-2,-3, \ldots$, for $j=1,2,3, \ldots, s$, is defined as

$$
\begin{aligned}
& { }_{q} F_{s}\left(\alpha_{1}, \ldots, \alpha_{q} ; \beta_{1}, \ldots, \beta_{s} ; z\right)=\sum_{n=0}^{\infty} \frac{\left(\alpha_{1}\right)_{n} \cdots\left(\alpha_{q}\right)_{n}}{\left(\beta_{1}\right)_{n} \cdots\left(\beta_{s}\right)_{n} n !} z^{n} \\
& \quad=1+\sum_{n=1}^{\infty} \frac{\left(\alpha_{1}\right)_{n} \cdots\left(\alpha_{q}\right)_{n}}{\left(\beta_{1}\right)_{n} \cdots\left(\beta_{s}\right)_{n}} \frac{z^{n}}{(n) !},
\end{aligned}
$$

with $q \leq s+1, q, s \in \mathbb{N}_{0}=\mathbb{N} \cup\{0\}$, and $(\alpha)_{n}$ is the well-known Pochhammer symbol related to the factorial and the Gamma function by the relation

$$
(\alpha)_{n}=\frac{(\alpha+n-1) !}{(\alpha-1) !}=\frac{\Gamma(\alpha+n)}{\Gamma(\alpha)} .
$$

Also (5) implies

$$
\begin{aligned}
& h_{p}\left(\alpha_{1}, \ldots, \alpha_{q} ; \beta_{1}, \ldots, \beta_{s} ; z\right) \\
& \quad=z^{-p}{ }_{q} F_{s}\left(\alpha_{1}, \ldots, \alpha_{q} ; \beta_{1}, \ldots, \beta_{s} ; z\right) \in M_{p} .
\end{aligned}
$$

Liu and Srivastava [2] defined a linear operator for functions belonging to the class of multivalent meromorphic function $H_{p}\left(\alpha_{1}, \ldots, \alpha_{q} ; \beta_{1}, \ldots, \beta_{s}\right): M_{p} \rightarrow M_{p}$ as follows:

$$
\begin{aligned}
H_{p} & \left(\alpha_{1}, \ldots, \alpha_{q} ; \beta_{1}, \ldots, \beta_{s}\right) \\
& =h_{p}\left(\alpha_{1}, \ldots, \alpha_{q} ; \beta_{1}, \ldots, \beta_{s} ; z\right) \star f(z) .
\end{aligned}
$$

If we assume for brevity that $H_{p, q, s}\left(\alpha_{1}\right)=H_{p}\left(\alpha_{1}, \ldots, \alpha_{q}\right.$; $\left.\beta_{1}, \ldots, \beta_{s}\right)$ then the following identity holds for this operator:

$$
\begin{aligned}
z\left[H_{p, q, s}\left(\alpha_{1}\right) f(z)\right]^{\prime}= & \alpha_{1} H_{p, q, s}\left(\alpha_{1}+1\right) f(z) \\
& -\left(\alpha_{1}+p\right) H_{p, q, s}\left(\alpha_{1}\right) f(z) .
\end{aligned}
$$

Shareef [3] defined and studied subclass $M Q_{p}\left(k, \lambda, \alpha_{1}\right)$ of meromorphic function associated with conic domain, for $k \geq$ $0,0 \leq \lambda<1$, and $p \geq 1$, as follows:

$$
\begin{aligned}
& -\frac{1}{p}\left[\frac{z\left(H_{p, q, s}\left(\alpha_{1}\right) f(z)\right)^{\prime}+\lambda z^{2}\left(H_{p, q, s}\left(\alpha_{1}\right) f(z)\right)^{\prime \prime}}{(1-\lambda) H_{p, q, s}\left(\alpha_{1}\right) f(z)+\lambda z\left(H_{p, q, s}\left(\alpha_{1}\right) f(z)\right)^{\prime}}\right] \\
& \quad \prec q_{k, \gamma}(z) .
\end{aligned}
$$

We now define a new subclass $M Q_{p}\left(b, k, \lambda, \alpha_{1}\right)$ of meromorphic function associated with conic domain, for $k \geq 0$, $0 \leq \lambda<1, p \geq 1$, and $b \geq 1$, as follows:

$$
\begin{aligned}
& -\frac{1}{p}[1 \\
& +\frac{1}{b}\left(\frac{z\left(H_{p, q, s}\left(\alpha_{1}\right) f(z)\right)^{\prime}+\lambda z^{2}\left(H_{p, q, s}\left(\alpha_{1}\right) f(z)\right)^{\prime \prime}}{(1-\lambda) H_{p, q, s}\left(\alpha_{1}\right) f(z)+\lambda z\left(H_{p, q, s}\left(\alpha_{1}\right) f(z)\right)^{\prime}}\right) \\
& -b] \prec q_{k, \gamma}(z) .
\end{aligned}
$$

Since $q_{k, \gamma}$ is a convex and univalent function, for $h(z) \prec$ $q_{k, \gamma}(z)$ it means $h\left(E^{*}\right)$ is contained in $q_{k, \gamma}\left(E^{*}\right)$, where

$$
\begin{aligned}
& h(z)=-\frac{1}{p}[1 \\
& \quad+\frac{1}{b}\left(\frac{z\left(H_{p, q, s}\left(\alpha_{1}\right) f(z)\right)^{\prime}+\lambda z^{2}\left(H_{p, q, s}\left(\alpha_{1}\right) f(z)\right)^{\prime \prime}}{(1-\lambda) H_{p, q, s}\left(\alpha_{1}\right) f(z)+\lambda z\left(H_{p, q, s}\left(\alpha_{1}\right) f(z)\right)^{\prime}}\right) \\
& -b] .
\end{aligned}
$$

In the next two sections, for brevity, we drop the subscripts of the operator $H_{p, q, s}\left(\alpha_{1}\right)$.

\section{Preliminary Results}

Lemma 1 (see [4]). Let $h_{2}(z)$ be convex in $E$ and $\Re\left(\lambda h_{2}(z)+\right.$ $\mu)>0$, where $\mu \in \mathbb{C}, \lambda \in \mathbb{C} \backslash\{0\}$, and $z \in E$. If $h_{1}(z)$ is analytic in $E$, with $h_{1}(0)=h_{2}(0)$, then

$$
\begin{aligned}
h_{1}(z)+\frac{z h_{1}^{\prime}(z)}{\lambda h_{1}(z)+\mu} & \prec h_{2}(z) \\
\text { implies } h_{1}(z) & \prec h_{2}(z) .
\end{aligned}
$$

Lemma 2 (see [5]). Let $h(z)=1+\sum_{n=1}^{\infty} c_{n} z^{n}$ and $H(z)=$ $1+\sum_{n=1}^{\infty} d_{n} z^{n}$ and $h \prec H$. If $H(z)$ is univalent and convex in $E$, then

$$
\left|c_{n}\right| \leq\left|d_{1}\right|
$$

for $n \geq 1$.

Lemma 3 (see [6]). If $q_{k, \gamma}(z)=1+q_{1} z+q_{2} z^{2}+\cdots$ then

$$
q_{1}= \begin{cases}\frac{8(1-\gamma)(\arccos (k))^{2}}{\pi^{2}\left(1-k^{2}\right)}, & 0<k<1, \\ \frac{8(1-\gamma)}{\pi^{2}}, & k=1, \\ \frac{\pi^{2}(1-\gamma)}{4 \sqrt{t}\left(k^{2}-1\right) k^{2}(t)(1+t)}, & k>1 .\end{cases}
$$

One now states and proves the main results.

\section{Main Results}

In this section we explore some of the geometric properties exhibited by the class $M Q_{p}\left(b, k, \lambda, \alpha_{1}\right)$.

We begin by discussing an inclusion property for the class $M Q_{p}\left(b, k, \lambda, \alpha_{1}\right)$.

Theorem 4. If $\mathfrak{R}\left(\alpha_{1}\right)>p \Re\left(b q_{k, \lambda}(z)-1\right)$ then

$$
M Q_{p}\left(b, k, \lambda, \alpha_{1}+1\right) \subset M Q_{p}\left(b, k, \lambda, \alpha_{1}\right) .
$$


Proof. Let $f \in M Q_{p}\left(b, k, \lambda, \alpha_{1}+1\right)$ and set

$$
\begin{aligned}
& -\frac{1}{p}[1 \\
& +\frac{1}{b}\left(\frac{z\left(H\left(\alpha_{1}\right) f(z)\right)^{\prime}+\lambda z^{2}\left(H\left(\alpha_{1}\right) f(z)\right)^{\prime \prime}}{(1-\lambda) H\left(\alpha_{1}\right) f(z)+\lambda z\left(H\left(\alpha_{1}\right) f(z)\right)^{\prime}}\right) \\
& -b]=h(z) .
\end{aligned}
$$

But differentiating (9) with respect to $z$ we get

$$
\begin{aligned}
& {\left[z H\left(\alpha_{1}\right) f(z)\right]^{\prime}} \\
& \quad=\alpha_{1} H\left(\left(\alpha_{1}+1\right) f(z)\right)^{\prime}
\end{aligned}
$$

$$
\begin{gathered}
-\left(\alpha_{1}+p\right)\left(H\left(\alpha_{1}\right) f(z)\right)^{\prime}, \\
z\left[H\left(\alpha_{1}\right) f(z)\right]^{\prime \prime}+\left[H\left(\alpha_{1}\right) f(z)\right]^{\prime} \\
=\alpha_{1} H\left(\left(\alpha_{1}+1\right) f(z)\right)^{\prime} \\
\quad-\left(\alpha_{1}+p\right)\left(H\left(\alpha_{1}\right) f(z)\right)^{\prime}, \\
z^{2}\left(H\left(\alpha_{1}\right) f(z)\right)^{\prime \prime} \\
=\alpha_{1} z\left(H\left(\alpha_{1}+1\right) f(z)\right)^{\prime} \\
-\left(\alpha_{1}+p+1\right) z\left(H\left(\alpha_{1}\right) f(z)\right)^{\prime} .
\end{gathered}
$$

Putting (18) in (17) we have

$$
\begin{aligned}
\frac{\lambda z \alpha_{1}(H(\alpha+1) f(z))^{\prime}+\left[1-\lambda\left(\alpha_{1}+p+1\right)\right] z\left(H\left(\alpha_{1}\right) f(z)\right)^{\prime}}{(1-\lambda) H\left(\alpha_{1}\right) f(z)+\lambda z\left(H\left(\alpha_{1}\right) f(z)\right)^{\prime}} & =-b p h(z), \\
\frac{\lambda \alpha_{1} z\left(H\left(\alpha_{1}+1\right) f(z)\right)^{\prime}+(1-\lambda) \alpha_{1}\left(H\left(\alpha_{1}+1\right) f(z)\right)}{(1-\lambda) H\left(\alpha_{1}\right) f(z)+\lambda z\left(H\left(\alpha_{1}\right) f(z)\right)^{\prime}} & =-b p h(z)+\left(\alpha_{1}+p\right) .
\end{aligned}
$$

Taking logarithmic derivative of (19) we have

$$
\begin{aligned}
& \frac{z\left(H\left(\alpha_{1}+1\right) f(z)\right)^{\prime}+\lambda z^{2}\left(H\left(\alpha_{1}+1\right) f(z)\right)^{\prime \prime}}{(1-\lambda)\left(H\left(\alpha_{1}+1\right) f(z)\right)+\lambda z\left(H\left(\alpha_{1}+1\right) f(z)\right)^{\prime}} \\
& \quad+b p h(z)+b(b-1)=\frac{-b p z h^{\prime}(z)}{-b p h(z)+\alpha_{1}+p}, \\
& \frac{z\left(H\left(\alpha_{1}+1\right) f(z)\right)^{\prime}+\lambda z^{2}\left(H\left(\alpha_{1}+1\right) f(z)\right)^{\prime \prime}}{(1-\lambda)\left(H\left(\alpha_{1}+1\right) f(z)\right)+\lambda z\left(H\left(\alpha_{1}+1\right) f(z)\right)^{\prime}} \\
& \quad=-b p\left[h(z)+\frac{z h^{\prime}(z)}{-b p h(z)+\alpha_{1}+p}\right]-b(b-1), \\
& -\frac{1}{p}[1 \\
& +\frac{1}{b}\left(\frac{z\left(H\left(\alpha_{1}+1\right) f(z)\right)^{\prime}+\lambda z^{2}\left(H\left(\alpha_{1}+1\right) f(z)\right)^{\prime \prime}}{(1-\lambda) H\left(\alpha_{1}+1\right) f(z)+\lambda z\left(H\left(\alpha_{1}+1\right) f(z)\right)^{\prime}}\right) \\
& -b]=h(z)+\frac{z h^{\prime}(z)}{-b p h(z)+\left(\alpha_{1}+p\right)} .
\end{aligned}
$$

Since $f \in M Q_{q}\left(b, k, \lambda, \alpha_{1}+1\right)$, therefore

$$
h(z)+\frac{z h^{\prime}(z)}{-b p h(z)+\left(\alpha_{1}+p\right)} \prec q_{k, \gamma}(z) .
$$

Using Lemma 2 we have

$$
h(z) \prec q_{k, \gamma}(z),
$$

provided $\mathfrak{R}\left(-b p q_{k, \lambda}(z)+\alpha_{1}+p\right)>0$ or equivalently $\mathfrak{R}\left(\alpha_{1}\right)>$ $p \Re\left(b q_{k, \gamma}(z)-1\right)$. Hence, $f \in M Q_{p}\left(b, k, \lambda, \alpha_{1}\right)$.

We now show that the class $M Q_{p}\left(b, k, \lambda, \alpha_{1}\right)$ is closed under a certain integral.

Theorem 5. If $f(z) \in M Q_{p}\left(b, k, \lambda, \alpha_{1}\right)$, then the integral

$$
B_{\eta}(H(\alpha) f(z))=\frac{\eta-p}{z^{\eta}} \int_{0}^{z} t^{\eta-1}(H(\alpha) f(z)) \mathrm{d} t
$$

maps $f(z)$ into $M Q_{p}\left(b, k, \lambda, \alpha_{1}\right)$.

Proof. From (23) we have

$$
z^{\eta} B_{\eta}(H(\alpha) f(z))=(\eta-p) \int_{0}^{z} t^{\eta-1}(H(\alpha) f(z)) \mathrm{d} t .
$$

Note that

$$
B_{\eta}(H(\alpha) f(z))=(H(\alpha)) B_{\eta} f(z) .
$$

Differentiating (24) above we get

$$
\begin{gathered}
\eta z^{\eta-1} B_{\eta}(H(\alpha) f(z))+z\left(B_{\eta}(H(\alpha) f(z))\right)^{\prime} \\
=(\eta-p) z^{\eta-1}(H(\alpha) f(z))
\end{gathered}
$$




$$
\begin{aligned}
\eta B_{\eta} & (H(\alpha) f(z))+z\left(B_{\eta}(H(\alpha) f(z))\right)^{\prime} \\
& =(\eta-p)(H(\alpha) f(z))
\end{aligned}
$$

Now let

6)

$$
-\frac{1}{p}[1
$$

Differentiate again

$$
\begin{aligned}
\eta( & \left.B_{\eta}(H(\alpha) f(z))\right)^{\prime}+z\left(B_{\eta}(H(\alpha) f(z))\right)^{\prime \prime} \\
& +\left(B_{\eta}(H(\alpha) f(z))\right)^{\prime}=(\eta-p)(H(\alpha) f(z))^{\prime}, \\
z & \left(B_{\eta}(f(z))\right)^{\prime \prime}=(\eta-p)\left(B_{\eta}(H(\alpha) f(z))\right)^{\prime} \\
& -(\eta+1)\left(B_{\eta}(f(z))\right)^{\prime} .
\end{aligned}
$$

$$
\begin{aligned}
& \frac{z B_{\eta}\left(H\left(\alpha_{1}\right) f\right)^{\prime}+\lambda\left[z(\eta-p)\left(H\left(\alpha_{1}\right) f\right)^{\prime}-z(1+\eta)\left(B_{\eta}\left(H\left(\alpha_{1}\right) f\right)\right)^{\prime}\right]}{(1-\lambda) B_{\eta}\left(H\left(\alpha_{1}\right) f\right)+\lambda z\left(B_{\eta}\left(H\left(\alpha_{1}\right) f\right)\right)^{\prime}}=-b p g(z)+b^{2}-b, \\
& \frac{\lambda z(\eta-p)\left(H\left(\alpha_{1}\right) f\right)^{\prime}+[1-\lambda(1+\eta)]\left[(\eta-p)\left(H\left(\alpha_{1}\right) f\right)-\eta\left(B_{\eta}\left(H\left(\alpha_{1}\right) f\right)\right)\right]}{(1-\lambda) B_{\eta}\left(H\left(\alpha_{1}\right) f\right)+\lambda z\left(B_{\eta}\left(H\left(\alpha_{1}\right) f\right)\right)^{\prime}}=-b p g(z)+b^{2}-b, \\
& \frac{(\eta-p)\left[\lambda z\left(H\left(\alpha_{1}\right) f\right)^{\prime}-(1-\lambda)\left(H\left(\alpha_{1}\right) f\right)\right]-\lambda \eta z\left(B_{\eta}\left(H\left(\alpha_{1}\right) f\right)\right)^{\prime}-(1-\lambda) \eta\left(B_{\eta}\left(H\left(\alpha_{1}\right) f\right)\right)}{(1-\lambda) B_{\eta}\left(H\left(\alpha_{1}\right) f\right)+\lambda z\left(B_{\eta}\left(H\left(\alpha_{1}\right) f\right)\right)^{\prime}}=-b p g(z)+b^{2}-b, \\
& \frac{(\eta-p)\left[\lambda z\left(H\left(\alpha_{1}\right) f\right)^{\prime}-(1-\lambda)\left(H\left(\alpha_{1}\right) f\right)\right]}{(1-\lambda) B_{\eta}\left(H\left(\alpha_{1}\right) f\right)+\lambda z\left(B_{\eta}\left(H\left(\alpha_{1}\right) f\right)\right)^{\prime}}=-b p g(z)+b^{2}-b+\eta .
\end{aligned}
$$

Now taking logarithmic derivative we have

$$
\begin{aligned}
& \frac{(\eta-p)\left[\lambda z\left(H\left(\alpha_{1}\right) f\right)^{\prime \prime}+\lambda\left(H\left(\alpha_{1}\right) f\right)^{\prime}+(1-\lambda)\left(H\left(\alpha_{1}\right) f\right)^{\prime}\right]}{(\eta-p)\left[\lambda z\left(H\left(\alpha_{1}\right) f\right)^{\prime}-(1-\lambda)\left(H\left(\alpha_{1}\right) f\right)\right]} \\
& -\frac{(1-\lambda) B_{\eta}\left(H\left(\alpha_{1}\right) f\right)^{\prime}+\lambda z\left(B_{\eta}\left(H\left(\alpha_{1}\right) f\right)\right)^{\prime \prime}+\lambda\left(B_{\eta}\left(H\left(\alpha_{1}\right) f\right)\right)^{\prime}}{(1-\lambda) B_{\eta}\left(H\left(\alpha_{1}\right) f\right)+\lambda z\left(B_{\eta}\left(H\left(\alpha_{1}\right) f\right)\right)^{\prime}}=\frac{-b p g^{\prime}(z)}{-b p g(z)+b^{2}-b}, \\
& -\frac{1}{p}\left[1+\frac{1}{b}\left(\frac{z\left(H\left(\alpha_{1}\right) f\right)^{\prime}+\lambda z^{2}\left(H\left(\alpha_{1}\right) f\right)^{\prime \prime}}{(1-\lambda)\left(H\left(\alpha_{1}\right) f\right)+\lambda z\left(H\left(\alpha_{1}\right) f\right)^{\prime}}\right)-b\right]=g(z)+\frac{z g^{\prime}(z)}{-b p g(z)+b^{2}-b} .
\end{aligned}
$$

Using Lemma 2 we get $g(z) \prec q_{k, \gamma}(z)$ which implies

$$
B_{\eta}(f(z)) \in M Q_{p}\left(b, k, \lambda, \alpha_{1}\right) .
$$

This proves the assertion.

Now we get coefficient estimates of the class $M Q_{p}(b, k$, $\left.\lambda, \alpha_{1}\right)$.
Theorem 6. If $f(z) \in M Q_{p}\left(b, k, \lambda, \alpha_{1}\right)$ and $f(z)$ is given by (1) then

$$
\left|a_{n}\right| \leq\left|\frac{b p q_{1}}{b(1-b)+b p-p+n}\right| \sum_{k=0}^{n-1}\left|a_{k}\right|, \quad a_{0}=1
$$

\section{provided}

$$
\begin{aligned}
& 2(\lambda p-1)+(3-n) \lambda<k \lambda \\
& \qquad \text { for } 1-\lambda p+(n-1) \lambda>0,
\end{aligned}
$$




$$
2(\lambda p-1)+(3-n) \lambda>k \lambda
$$$$
\text { for } 1-\lambda p+(n-1) \lambda<0 \text {, }
$$

for all $k \leq n$.

Proof. Let $f(z) \in M Q_{p}\left(b, k, \lambda, \alpha_{1}\right)$; then, by definition, we have

$$
\begin{aligned}
& -\frac{1}{p}[1 \\
& +\frac{1}{b}\left(\frac{z\left(H\left(\alpha_{1}\right) f(z)\right)^{\prime}+\lambda z^{2}\left(H\left(\alpha_{1}\right) f(z)\right)^{\prime \prime}}{(1-\lambda) H\left(\alpha_{1}\right) f(z)+\lambda z\left(H\left(\alpha_{1}\right) f(z)\right)^{\prime}}\right. \\
& -b)]=h(z) \prec q_{k, \gamma}(z), \\
& -\frac{z\left(H\left(\alpha_{1}\right) f(z)\right)^{\prime}+\lambda z^{2}\left(H\left(\alpha_{1}\right) f(z)\right)^{\prime \prime}}{p(1-\lambda) H\left(\alpha_{1}\right) f(z)+\lambda z\left(H\left(\alpha_{1}\right) f(z)\right)^{\prime}} \\
& =b h(z)+\frac{b}{p}-\frac{b^{2}}{p},
\end{aligned}
$$

$$
\begin{aligned}
f^{\prime \prime}(z)= & -p(-p-1) z^{-p-2} \\
& +\sum_{n=0}^{\infty} a_{n}(n-p)(n-p-1) z^{n-p-2}, \\
z^{2} f^{\prime \prime}(z)= & -p(-p-1) z^{-p} \\
& +\sum_{n=0}^{\infty} a_{n}(n-p)(n-p-1) z^{n-p} .
\end{aligned}
$$

Assuming $h(z)=1+\sum_{n=1}^{\infty} c_{n} z^{n}$, then (35) becomes

$$
\begin{aligned}
z^{-p} & -\sum_{n=0}^{\infty} a_{n} \frac{(n-p)}{p} z^{n-p}+\lambda\left(-(p+1) z^{-p}\right. \\
& \left.+\sum_{n=0}^{\infty} a_{n} \frac{(n-p)(n-p-1)}{p} z^{n-p}\right), \\
(1-\lambda p-\lambda) z^{-p} & \\
& -\sum_{n=1}^{\infty} a_{n} \frac{n-p}{p}[1+\lambda(n-p-1)] z^{n-p}=[b \\
& \left.+\sum_{n=1}^{\infty} b c_{n} z^{n}+\frac{b(1-b)}{p}\right]\left[(1-\lambda p-\lambda) z^{-p}\right. \\
& \left.+\sum_{n=1}^{\infty} a_{n}[1+\lambda(n-p-1)] z^{n-p}\right] .
\end{aligned}
$$

From (37) we have

$$
(1-\lambda p-\lambda) z^{-p}
$$

$$
\begin{aligned}
& -\sum_{n=1}^{\infty} a_{n} \frac{n-p}{p}[1+\lambda(n-p-1)] z^{n-p}=\left[b+b c_{1} z\right. \\
& \left.+b c_{2} z^{2}+b c_{3} z^{3}+\cdots+b c_{n} z^{n}+\cdots+\frac{b(1-b)}{p}\right] \\
& \cdot\left[(1-\lambda p-\lambda) z^{-p}\right.
\end{aligned}
$$

$$
\left.+\sum_{n=1}^{\infty} a_{n}[1+\lambda(n-p-1)] z^{n-p}\right] .
$$

Now comparing coefficients of $z^{1-p}$ we have

$$
\begin{aligned}
& -a_{1} \frac{1-p}{p}(1-\lambda p)=a_{1} b(1-\lambda p)+a_{1}\left(\frac{b(1-b)}{p}\right) \\
& \cdot(1-\lambda p)+b c_{1}(1-\lambda p-\lambda), \\
& -a_{1}(1-\lambda p)\left[\frac{b(1-b)+b p-p+1}{p}\right] \\
& =b c_{1}(1-\lambda p-\lambda),
\end{aligned}
$$

$a_{1}=-\frac{p}{(1-\lambda p)[b(1-b)+b p-p+1]}(1-\lambda p-\lambda)$

$$
\cdot b c_{1}
$$


and comparing the coefficients of $z^{2-p}$ gives

$$
\begin{aligned}
& -a_{2}\left[\frac{2-p}{p}\right][1+\lambda(1-p)]=a_{2}[1+\lambda(1-p)][b \\
& \left.+\frac{b(1-b)}{p}\right]+a_{1}[1-\lambda p] b c_{1}+b c_{2}(1-\lambda p-\lambda) \\
& -a_{2}[1+\lambda(1-p)]\left[\frac{2-p}{p}+\frac{b p+b(1-b)}{p}\right]=a_{1}[1 \\
& -\lambda p] b c_{1}+b c_{2}(1-\lambda p-\lambda), \\
& -a_{2}[1+\lambda(1-p)]\left[\frac{b(1-b)+b p-p+2}{p}\right]=a_{1}[1 \\
& -\lambda p] b c_{1}+b c_{2}(1-\lambda p-\lambda), \\
& a_{2}=-\frac{p}{(1+\lambda(1-p))(b(1-b)+b p-p+2)}((1 \\
& \left.-\lambda p-\lambda) b c_{2}+[1-\lambda p] b a_{1} c_{1}\right)
\end{aligned}
$$

and for the coefficient of $z^{3-p}$ we have

$$
\begin{aligned}
- & a_{3}[1+\lambda(2-p)]\left[\frac{b(1-b)+b p-p+3}{p}\right] \\
& =b c_{1} a_{2}[1+\lambda(1-p)]+b c_{2} a_{1}[1-\lambda p]+b c_{3}[1 \\
& -\lambda p-\lambda], \\
a_{3} & =-\frac{p}{(1+\lambda(2-p))(b(1-b)+b p-p+3)}([1 \\
& -\lambda p-\lambda] b c_{3}+[1-\lambda p] b c_{2} a_{1}+[1+\lambda(1-p)] \\
& \left.\cdot b c_{1} a_{2}\right),
\end{aligned}
$$

which generalise to

$$
\begin{aligned}
a_{n} & =-\frac{p}{(1-\lambda p+(n-1) \lambda)(b(1-b)+b p-p+n)}[(1 \\
& -\lambda p-\lambda) b c_{n}+(1-\lambda p) b c_{n-1} a_{1}+(1-\lambda p+\lambda) \\
& \left.\cdot b c_{n-2} a_{2}+\cdots+(1-\lambda p+(n-2) \lambda) b c_{1} a_{n-1}\right] .
\end{aligned}
$$

The above expression can also be written as

$$
\begin{aligned}
a_{n}= & -\frac{p}{(1-\lambda p+(n-1) \lambda)(b(1-b)+b p-p+n)} \\
& \cdot \sum_{k=1}^{n}[1-\lambda p+(k-2) \lambda] c_{n-k-1} a_{k-1},
\end{aligned}
$$

with $a_{0}=1$.

Now taking

$$
A_{n, k}=-\frac{1-\lambda p+(k-2) \lambda}{1-\lambda p+(n-1) \lambda}, \quad n \geq k,
$$

we have

$$
\begin{aligned}
& \left|A_{n, k}\right|<1, \\
& \text { if } \begin{cases}2(\lambda p-1)+(3-n) \lambda<k \lambda & \text { for } 1-\lambda p+(n-1) \lambda>0, \\
2(\lambda p-1)+(3-n) \lambda>k \lambda & \text { for } 1-\lambda p+(n-1) \lambda<0,\end{cases}
\end{aligned}
$$

for all $n \geq k$. Since $q_{k, \gamma}(z)$ is univalent and $q_{k, \gamma}(E)$ is convex, applying Rogosinski's theorem we have

$$
\left|c_{n}\right| \leq\left|q_{1}\right|
$$

where $q_{1}$ is given in (15). Under the conditions given in (45), expressions (39)-(42) give

$$
\begin{aligned}
& \left|a_{1}\right| \leq\left|\frac{b p q_{1}}{b(1-b)+b p-p+1}\right|, \\
& \left|a_{2}\right| \leq\left|\frac{b p q_{1}}{b(1-b)+b p-p+2}\right|\left(1+\left|a_{1}\right|\right), \\
& \left|a_{3}\right| \leq\left|\frac{b p q_{1}}{b(1-b)+b p-p+3}\right|\left(1+\left|a_{1}\right|+\left|a_{2}\right|\right),
\end{aligned}
$$

$$
\begin{aligned}
&\left|a_{n}\right| \leq\left|\frac{b p q_{1}}{b(1-b)+b p-p+n}\right| \\
& \cdot\left(1+\left|a_{1}\right|+\left|a_{2}\right|+\cdots+\left|a_{n-1}\right|\right) .
\end{aligned}
$$

This can also be written as

$$
\left|a_{n}\right| \leq\left|\frac{b p q_{1}}{b(1-b)+b p-p+n}\right| \sum_{k=0}^{n-1}\left|a_{k}\right| .
$$

This concludes the proof.

\section{Competing Interests}

The authors declare that there is no conflict of interests regarding the publication of this paper.

\section{Acknowledgments}

The work here is supported by AP-2013-009.

\section{References}

[1] S. Kanas and A. Wisniowska, "Conic domains and k-starlike functions," Revue Roumaine de Mathematique Pures et Appliquees, vol. 45, no. 4, pp. 647-657, 2000.

[2] J.-L. Liu and H. M. Srivastava, "Classes of meromorphically multivalent functions associated with the generalized hypergeometric function," Mathematical and Computer Modelling, vol. 39, no. 1, pp. 21-34, 2004.

[3] Z. Shareef, Some geometric properties of certain classes of analytic functions [Ph.D. thesis], Universiti Kebangsaan Malaysia, Bangi, Malaysia, 2015.

[4] P. Eenigenburg, P. T. Mocanu, S. S. Miller, and M. O. Reade, "On a briot-bouquet differential subordination," in General Inequalities, vol. 3 of International Series of Numerical Mathematics, pp. 339-348, Birkhäuser, Basel, Switzerland, 1983. 
[5] W. Rogosinski, "On the coefficients of subordinate functions," Proceedings of the London Mathematical Society, Series 2, vol. 48, no. 1, pp. 48-82, 1945.

[6] F. M. Al-Oboudi and K. A. Al-Amoudi, "On classes of analytic functions related to conic domains," Journal of Mathematical Analysis and Applications, vol. 339, no. 1, pp. 655-667, 2008. 


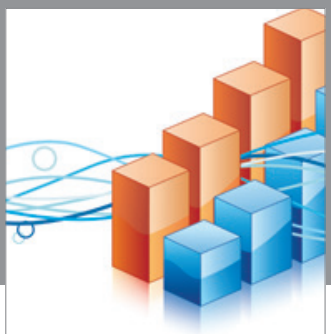

Advances in

Operations Research

vatem alat4

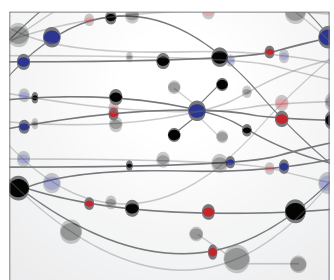

\section{The Scientific} World Journal
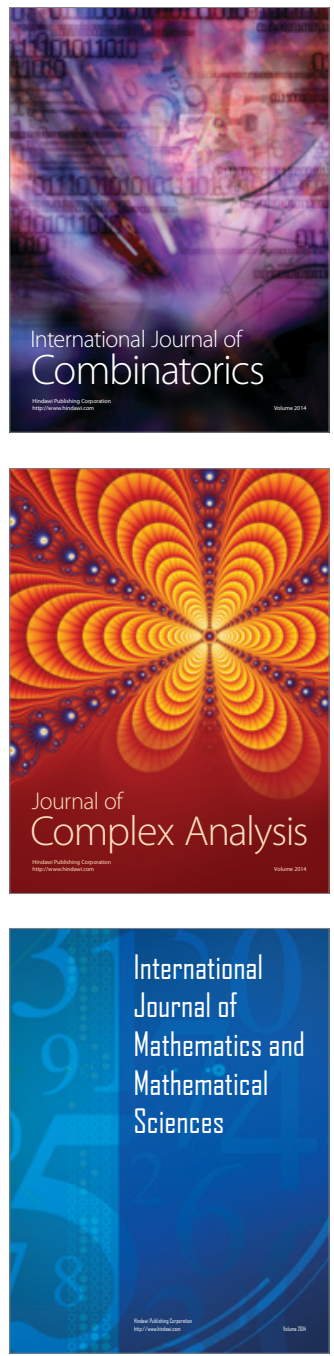
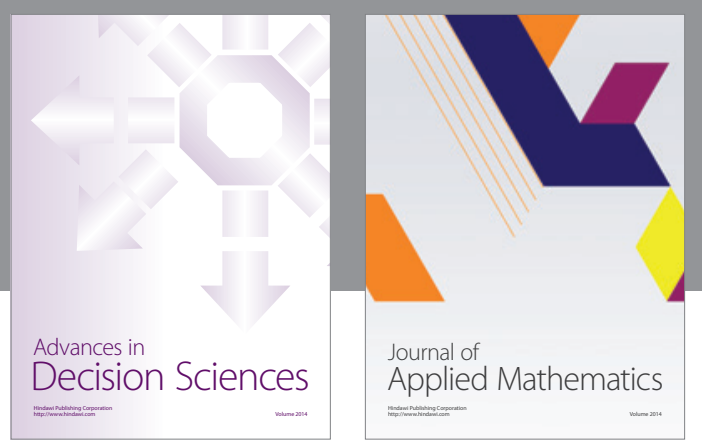

Algebra

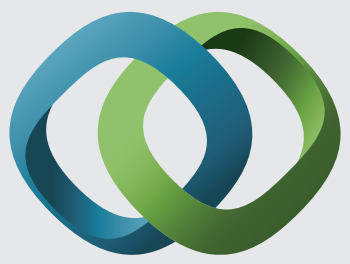

\section{Hindawi}

Submit your manuscripts at

http://www.hindawi.com
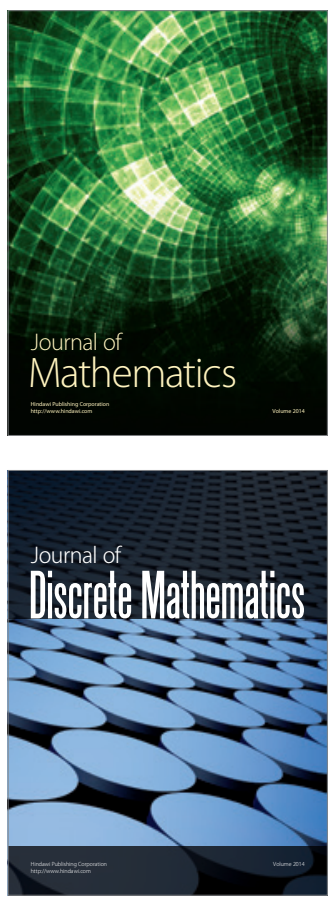

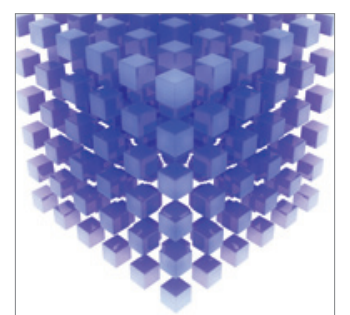

Mathematical Problems in Engineering
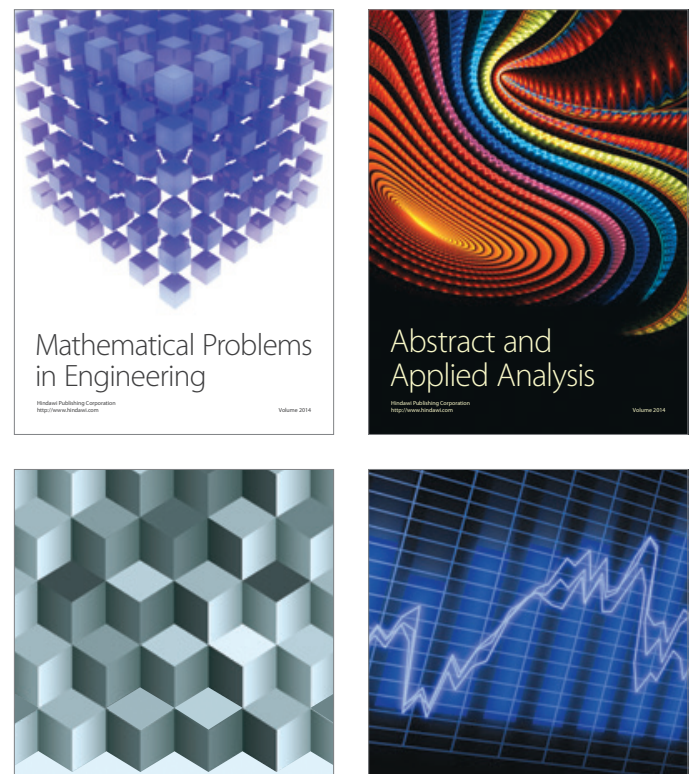

Journal of

Function Spaces

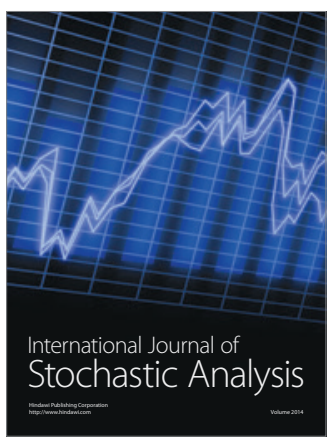

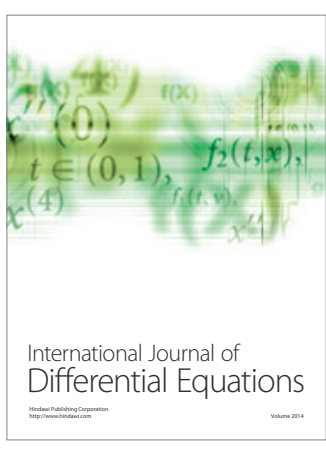
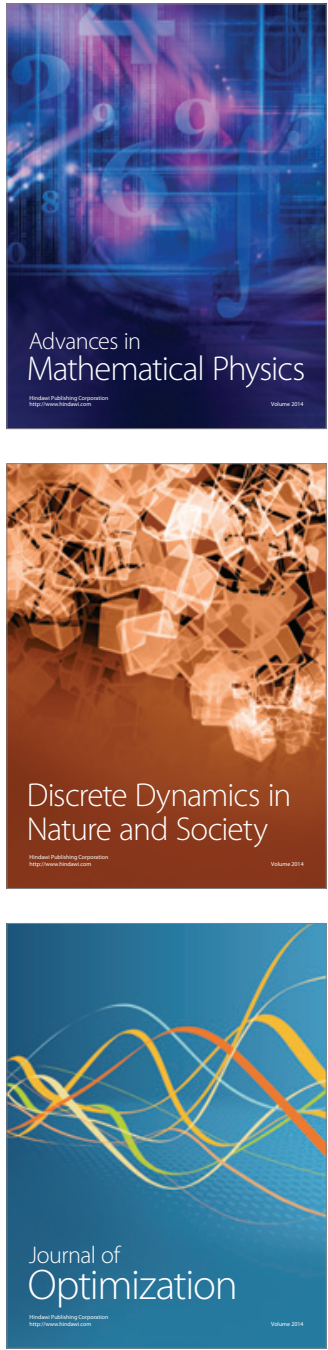